\title{
The sniffing position provides greater occipito- atlanto-axial angulation than simple head extension: a radiological study
}

\author{
[La position de reniflement fournit une meilleure angulation occipito-atlaïdo- \\ axiale que la simple extension de la tête: une étude radiologique]
}

Ichiro Takenaka MD, Kazuyoshi Aoyama MD, Tamao Iwagaki MD, Hiroshi Ishimura MD, Tatsuo Kadoya MD

Purpose: While the anatomic sniffing position has traditionally been considered the standard head and neck position for laryngoscopy, recent evidence suggests that the sniffing position provides no significant advantage over simple head extension. To establish if the sniffing position provides an anatomic advantage, we compared the occipito-atlanto-axial extension angle, a key determinant for obtaining a good laryngeal view during laryngoscopy, in simple head extension and sniffing positions.

Methods: Thirty volunteers with normal cervical spines were studied. Radiological examinations of the lateral cervical spine were taken and compared in each of the following three positions for each subject: neutral position (flat on the table with no pillow and without head extension or flexion); simple head extension (head maximally extended without a pillow); and the sniffing position (head extension with cervical flexion obtained by $7 \mathrm{~cm}$ occipital elevation).

Results: Mean angles of the occipito-atlanto-axial extension in simple head extension and the sniffing position were $20.4^{\circ} \pm$ $5.1^{\circ}$ and $24.2^{\circ} \pm 5.6^{\circ}$, respectively $(P<0.01)$.

Conclusion: The anatomic sniffing position provides greater occipito-atlanto-axial extension compared to simple head extension. These findings should be taken into consideration when optimizing patient positioning for laryngoscopy.

CAN J ANESTH $2007 / 54: 2 /$ pp 129-133
Objectif: La position anatomique de reniflement est traditionnellement considérée comme la position standard de la tête et du cou pour la laryngoscopie; toutefois, des études récentes suggèrent que cette position ne présente aucun avantage significatif par rapport à la simple extension de la tête. Nous avons comparé l'angle d'extension occipito-atlaïdo-axial dans les positions de simple extension de la tête et de reniflement afin de déterminer si la position de reniflement présentait un avantage anatomique, l'angle étant un élément-clé dans l'obtention d'une bonne vision laryngée pendant la laryngoscopie.

Méthode : L'étude portait sur trente volontaires avec colonnes cervicales normales. Chaque sujet a subi des examens radiologiques de la colonne cervicale latérale dans les trois positions suivantes: position neutre (à plat sur la table sans oreiller et sans extension ou flexion de la tête) ; extension simple de la tête (tête en extension maximale sans oreiller); et position de reniflement (extension de la tête avec flexion cervicale obtenue par une élévation occipitale de $7 \mathrm{~cm}$ ). Les résultats ont ensuite été comparés.

Résultats : Les angles moyens d'extension occipito-atlaïdo-axiale dans l'extension simple de la tête et la position de reniflement étaient de $20,4^{\circ} \pm 5,1^{\circ}$ et $24,2^{\circ} \pm 5,6^{\circ}$ respectivement $(P<$ 0,01).

Conclusion: La position anatomique de reniflement présente une plus grande extension occipito-atlaïdo-axiale que la simple extension de la tête. Ces résultats devraient être pris en compte pour obtenir un meilleur positionnement du patient lors de la laryngoscopie.

From the Department of Anesthesia, Nippon Steel Yawata Memorial Hospital, Kitakyushu, Japan.

Address correspondence to: Dr. Ichiro Takenaka, Department of Anesthesia, Nippon Steel Yawata Memorial Hospital, 1-1-1

Harunomachi, Yahatahigashi-ku, Kitakyushu 805-8508, Japan. Phone: 81-93-671-9420; Fax: 81-93-671-9605; E-mail: takenaka.i@ns. yawata-mhp.or.jp

Support was provided solely from institutional and/or departmental sources.

Accepted for publication September 18, 2006.

Revision accepted November 8, 2006. 
$\mathrm{P}$ LACING the head and neck in the sniffing position has been considered to be the most important maneuver for obtaining the good laryngeal view during conventional laryngoscopy. ${ }^{1-4}$ However, Adnet et al..$^{5}$ recently demonstrated that the sniffing position does not achieve alignment of the axes of the mouth, pharynx and larynx in awake patients. They subsequently demonstrated that the sniffing position provides no advantage for improving the laryngoscopic view compared to simple head extension. ${ }^{6}$

Direct laryngoscopy requires movement of the cervical spine for optimal visualization of the glottis. Among the cervical spine segments, extension at the occipito-atlanto-axial (OAA) complex is pivotal for several reasons. First, most extension created by direct laryngoscopy occurs at the OAA complex and the subaxial cervical segments are only minimally displaced during laryngoscopy. ${ }^{7}$ Second, Calder et al. ${ }^{8}$ have demonstrated that in patients with cervical spine disease involving the OAA complex that there is a higher prevalence of difficult laryngoscopy in comparison to patients with disease below the level of the axis. Third, OAA extension enhances mouth opening ability. ${ }^{9}$ Finally, aligning the pharyngeal axis with the axis of the mouth is the main factor for improving laryngoscopic view, and depends entirely on the degree of the OAA extension (Figure 1). ${ }^{10}$ Thus, greater OAA extension can produce a good laryngeal view during laryngoscopy.

Ordway et al. ${ }^{11}$ quantified the contribution of segmental motion of the cervical spine in four cervical end-range positions which included full-length head and neck extension, full-length flexion, protrusion, and retraction positions. These investigators found that the greatest extension at the OAA complex occurred in protrusion, and not in full-length extension. Protrusion positioning consists of extension at the OAA complex and flexion of the lower cervical spine, which is equal to the sniffing position. ${ }^{12} \mathrm{We}$ hypothesized that the sniffing position may provide greater OAA extension compared with simple head extension, allowing a good laryngeal view during laryngoscopy. To test this hypothesis, we examined maximal extension angle of the OAA complex in simple head extension and sniffing positions in healthy adult volunteers with normal cervical spines.

\section{Methods}

\section{Subjects}

Thirty healthy male volunteers without cervical spine pathology were studied. The study was approved by the Institutional Ethical Committee, and writ-

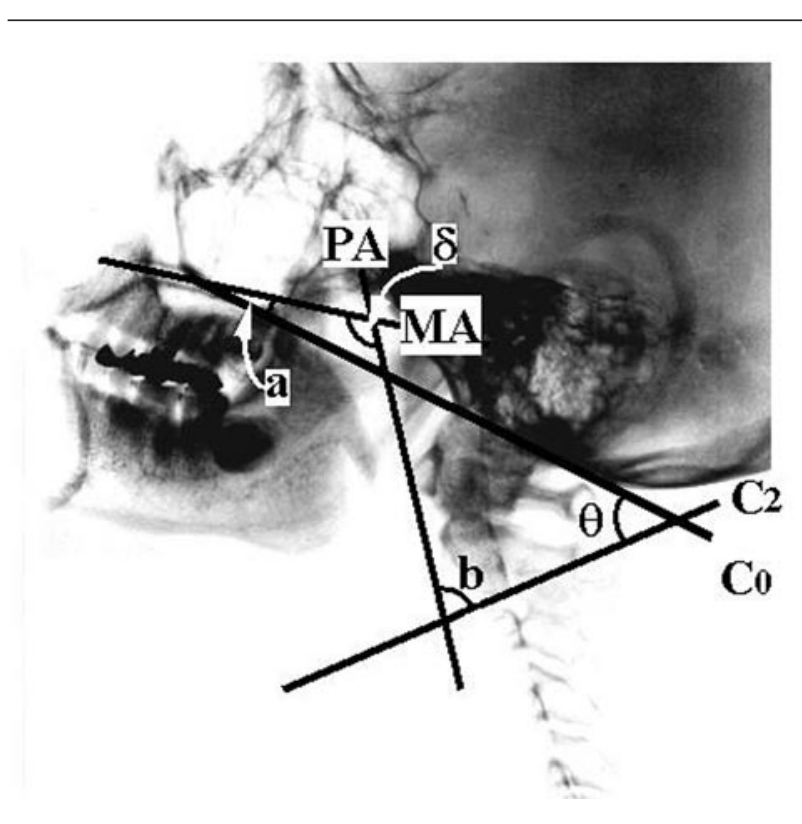

FIGURE 1 Cervical radiograph displaying the mouth axis (MA), the pharyngeal axis (PA), and reference lines for the occiput (C0) and the axis (C2). According to the definition described by Adnet et al., ${ }^{5}$ the MA and PA are defined by the line parallel to the hard palate and the line passing through the anterior portion of the atlas $(\mathrm{Cl})$ and of $\mathrm{C} 2$, respectively. Reference lines for $\mathrm{C} 0$ and $\mathrm{C} 2$ are defined as the McGregor line, which connects the dorsal edge of the hard palate and the most dorsal and caudal portion of $\mathrm{C} 0$, and the line passing through anterior and posterior basal plate of the $\mathrm{C} 2$ vertebral body, respectively. The angle between the MA and C0 (a) is constant despite changes in head position because both are fixed lines on the skull base. The angle between the PA and C2 (b) is also constant because the transverse atlantal ligament holds the odontoid process of $\mathrm{C} 2$ to the posterior surface of the anterior arch of $\mathrm{Cl}$. The angle between the MA and PA $(\delta)$, therefore, depends entirely upon the angle between $\mathrm{C} 0$ and $\mathrm{C} 2(\theta)$, as follows: $\delta=\theta+b-a$.

ten informed consent to participate in the study was obtained from all subjects.

\section{Experimental protocol}

The subjects lay on a flat table with no pillow, looking straight without head extension or flexion, which was defined as the neutral position. ${ }^{5}$ The subject was then asked to extend her/his head maximally without a pillow (defined as simple head extension) and with a pillow (the sniffing position) in randomized order (Figure 2). ${ }^{5}$ We used a solid platform $7 \mathrm{~cm}$ in height as the pillow to keep the degree of cervical flexion constant (Figure 2). The observer (I.T.) was careful 


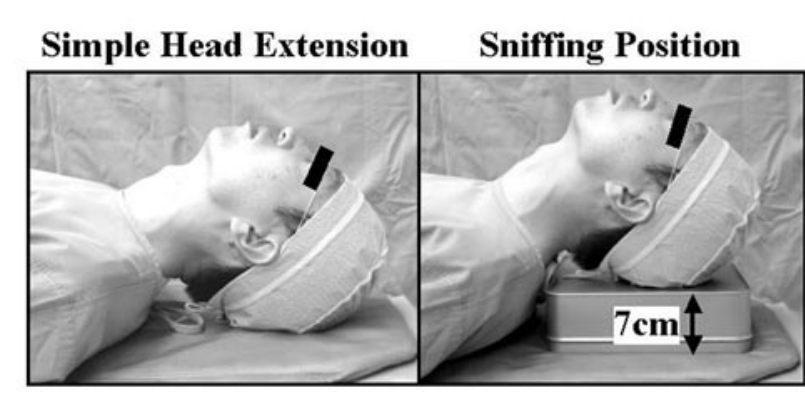

FIGURE 2 Sample photographs in simple head extension (head maximally extended without a pillow) and the sniffing position (head extension with cervical flexion introduced by using a rigid metal box $7 \mathrm{~cm}$ in height).

not to move the subject's shoulders when the head was extended. Simple head extension and the sniffing position were determined by actively positioning with the subject's effort. Lateral cervical radiographs were taken in all three positions.

\section{Radiological measurements}

Two experienced radiologists who were blinded to the purpose of the study analyzed the radiographs. As a reference for the position of the occiput $(\mathrm{C} 0)$ in relation to the cervical spine, the McGregor line was used (Figure 3). This line connected the most dorsal edge of, and caudal portion of the occiput and the dorsal edge of the hard palate. The reference line for the axis (C2) was drawn through the basal plate of the vertebral body (Figure 3). Because reference lines did not intersect on the radiograph in some subjects, the $\mathrm{C} 0$ or $\mathrm{C} 2$ angle was defined as the difference in angle between the $\mathrm{C} 0$ or $\mathrm{C} 2$ reference line and the common line which was the ventral vertical edge on each radiograph, respectively (Figure 3 ). The $\mathrm{C} 0-2$ angle was calculated by the difference between $\mathrm{C} 0$ and $\mathrm{C} 2$ angles. The OAA extension angle was defined as the difference in the $\mathrm{C} 0-2$ angle between the neutral position and simple head extension or the sniffing position.

\section{Statistical analysis}

Sample size was calculated based on our previous study, ${ }^{10}$ which demonstrated a standard deviation in the angle of the OAA extension of $4.7^{\circ}$ in volunteers with normal cervical spines. Assuming the SD in the OAA extension angle of $5^{\circ}$, a sample size of 26 would provide $80 \%$ power at the $5 \%$ significance level to

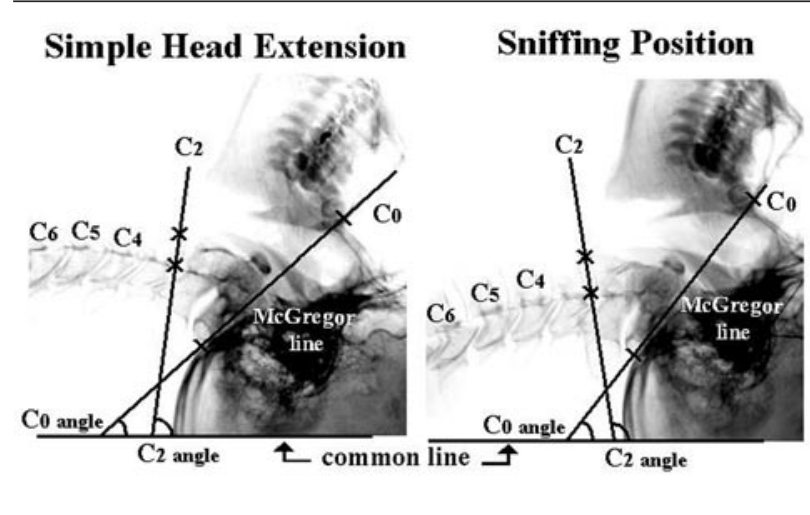

FIGURE 3 Representative lateral neck radiographs in simple head extension and sniffing positions. Note the difference in position of lower cervical spines in both positions. The McGregor line, which connects the most dorsal edge of and caudal portion of the occiput and the dorsal edge of the hard palate, is used as reference for the occiput (C0). A reference line for the axis (C2) is drawn through the basal plate of the vertebral body. The angle between each reference line and a common line that is the ventral vertical edge on each radiograph is measured. The $\mathrm{C} 0-2$ angle is calculated by the difference between $\mathrm{C} 0$ and $\mathrm{C} 2$ angles.

detect a difference of $4^{\circ}$ between simple head extension and sniffing positions. The difference in OAA extension angles between the two positions compared using a paired Student's $t$ test. A $P$ value $<0.05$ was considered significantly different.

\section{Results}

Demographics

Mean $( \pm S D)$ values of age, height, and weight of the 30 subjects were $32 \pm 8 \mathrm{yr}$ (range, $25-52 \mathrm{yr}$ ), and 171 $\pm 6 \mathrm{~cm}(162-180 \mathrm{~cm})$, and $67 \pm 12 \mathrm{~kg}(53-90 \mathrm{~kg})$, respectively.

\section{Occipito-atlanto-axial extension angle}

Mean angles of the OAA extension in simple head extension and sniffing positions were $20.4^{\circ} \pm 5.1^{\circ}$ and $24.2^{\circ} \pm 5.6^{\circ}$, respectively $(P<0.01)$. The sniffing position provided greater OAA extension compared with simple head extension. The difference in the OAA extension angle between both positions was $3.8^{\circ} \pm$ $3.7^{\circ}$. Lateral radiographs showed that near maximal extension of the OAA complex occurred in both positions in all subjects. The variation in the radiographic angle measurement was less than $1.2^{\circ}$, demonstrating low interobserver variability. 


\section{Discussion}

In awake volunteers with normal cervical spines, the OAA extension angle in simple head extension was $20.4^{\circ}$, which is similar to results from our previous study in adult patients. ${ }^{10}$ The sniffing position produced an OAA extension angle of $24.2^{\circ}$, and the difference between both positions was $3.8^{\circ}$. Ordway et al. ${ }^{11}$ found that the OAA extension angle in the protrusion (sniffing) position was significantly larger than that observed in full-length extension of the entire cervical spine, and the difference in angle was $8.3^{\circ}$. The principal difference when comparing full-length extension, simple head extension, and protrusion (sniffing) positions resides in the direction of movement of the lower cervical spine, which is extension, near neutral (or slight flexion), and flexion, respectively. This indicates that the degree of OAA extension increases gradually with flexion of the lower cervical spine. We consider a possible reason for this as follows. Cadaveric studies show that the OAA extension angle increased when the intersegmental ligamentous restraints were removed from the cervical spine, which appeared to result from the cup-like structure of the OAA articulation. ${ }^{13}$ When the lower cervical spine is in extension or a neutral position, these ligaments are stretched, which prevents motion of the OAA complex. In the sniffing position, these ligaments become loose as a result of flexion of the lower cervical spine, allowing for full extension of the articular surface in the OAA complex. That is, flexion of the lower cervical spine is needed for maximal extension of the OAA complex.

The three-axes alignment theory has been used to explain the steps in dynamic structural interactions during laryngoscopy, but Adnet et al. ${ }^{5}$ have clarified a contradiction of the theory using magnetic resonance imaging. In contrast, the obstacle theory proposed by Isono $^{14}$ is useful in understanding this discrepancy, but does not fully explain why the sniffing position confers an advantage for improving the laryngoscopic view. ${ }^{14}$ Our results suggest that there are two kinds of obstacles between the laryngoscopist's visual field and the glottis. One obstacle is located anterior to the oral airway, and the other is located posteriorly (Figure $4 \mathrm{~A})$. To visualize the glottis, the space between both obstacles must be enlarged (Figure 4B). In simple head extension, extension of the head (OAA complex) enlarges this space by moving the posterior obstacle downward, ensuring a posterior field of vision (Figure 4C). In the sniffing position, flexion of the neck (lower cervical spine) first moves both obstacles upwards without changing their relative positions (Figure 4D). Because the cervical flexion produces a

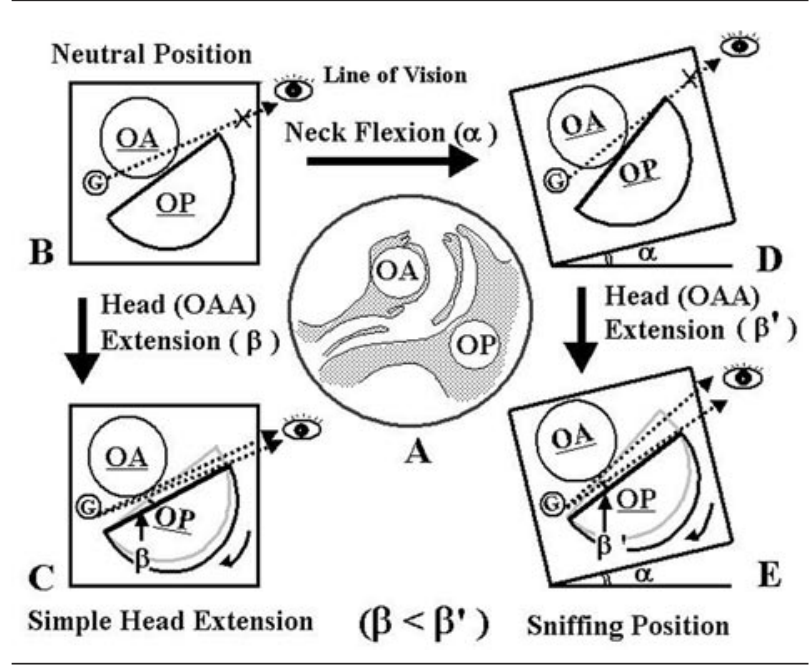

FIGURE 4 Theoretical explanation of the advantage of the sniffing position for laryngoscopy compared to simple head extension using the "obstacle theory". 14 There are two types of obstacles between laryngoscopist's visual field and the glottis. One obstacle is located anterior to the oral airway, and the other is that located posteriorly (A). To visualize the glottis under direct vision, the space between both obstacles must be enlarged (B). Application of head [occipito-atlanto-axial (OAA) complex] extension $(\beta)$ enlarges this space by moving the posterior obstacle downward, ensuring posterior field of vision (C). In the sniffing position, neck flexion $(\alpha)$ moves both obstacles upward without a change in their relative position (D). Because neck flexion produces a greater OAA extension $\left(\beta^{\prime}, \beta<\beta^{\prime}\right)$, a larger space between both obstacles is established $(\mathrm{E})$. OA = obstacle located anterior to the oral airway (tongue, mandible, and others); OP = obstacle located posterior to the airway (maxilla, teeth, and others); $\mathrm{G}=$ glottis; $\alpha=$ angle of cervical flexion; $\beta$ and $\beta^{\prime}=$ angles of OAA extension in simple head extension and the sniffing position, respectively.

greater degree of the OAA extension compared with simple head extension, a larger space between both obstacles is established (Figure 4E). Our results clarify part of the anatomic advantage of the sniffing position for laryngoscopy compared to simple head extension.

In contrast to our study, Adnet et al. ${ }^{6}$ demonstrated no differences in the laryngoscopic views and intubation difficulty scale comparisons between simple head extension and sniffing positions in 456 patients. Several anatomic factors contribute to visualization of the glottis during laryngoscopy, of which OAA extension is only one addition; a difference in the OAA extension angle between simple head extension and sniffing positions was only $3.8^{\circ}$. Thus, a large-scale study may be necessary to clarify that the anatomic 
advantage of the sniffing position favours laryngoscopic viewing in patients with documented or potentially difficult airways.

There are several potential limitations of our experimental design. First, we did not examine the relationship between the OAA extension angle and visualization of the larynx during direct laryngoscopy. Second, the maximal OAA extension angle would be underestimated because we did not use continuous fluoroscopy to assess the extension angle. Intra-subject variability may have existed in part, from the fact that the endpoint for extending the head maximally depended on the voluntary participation of each subject. We did not examine subjects to document maximal extension, because near maximal extension at the OAA complex was confirmed radiographically in both the simple head extension and sniffing positions. Third, we used a $7-\mathrm{cm}$ high rigid platform as a "pillow" to avoid the variable degree of cervical flexion caused by the compressibility of a regular pillow. However, use of a single standard pillow size did not always provide optimal cervical flexion for all subjects, because of modest variations in weight, head circumference, and length of the neck. Finally, we performed this study in volunteers with normal cervical spines and not in patients with limited head extension. Hence, our findings may not be generalizable to patients with disease of the cervical spine.

In conclusion, in comparison to simple head extension, the sniffing position provides greater OAA extension in awake volunteers, indicating that the sniffing position may confer a distinct anatomic advantage for laryngoscopy. These findings should be taken into consideration when optimizing patient positioning for laryngoscopy.

\section{References}

1 Horton WA, Faby L, Charters P. Defining a standard intubating position using "angle finder". Br J Anaesth 1989; 62: 6-12.

2 Benumof JL. Difficult laryngoscopy: obtaining the best view. (Editorial) Can J Anaesth 1994; 41: 361-5.

3 Benumof JL. Conventional (laryngoscopic) orotracheal and nasotracheal intubation (single-lumen tube). In: Benumof JL (Ed.). Airway Management: Principles and Practice, lst ed. St. Louis: Mosby; 1996: 261-76.

4 Gal TJ. Airway management. In: Miller RD (Ed.). Anesthesia, 6th ed. Philadelphia: Elsevier Churchill Livingstone; 2005: 1637-5.

5 Adnet F, Borron SW, Dumas JL, Lapostolle F, Cupa $M$, Lapandry C. Study of the "sniffing position" by magnetic resonance imaging. Anesthesiology 2001; 94: 83-6.
6 Adnet F, Baillard C, Borron SW, et al. Randomized study comparing the "sniffing position" with simple head extension for laryngoscopic view in elective surgery patients. Anesthesiology 2001; 95: 836-41.

7 Sawin PD, Todd MM, Traynelis VC, et al. Cervical spine motion with direct laryngoscopy and orotracheal intubation. An in vivo cinefluoroscopic study of subjects without cervical abnormality. Anesthesiology 1996; 85: 26-36.

8 Calder I, Calder J, Crockard HA. Difficult direct laryngoscopy in patients with cervical spine disease. Anaesthesia 1995; 50: 756-63.

9 Calder I, Picard J, Chapman M, O'Sullivan C, Crockard HA. Mouth opening: a new angle. Anesthesiology 2003; 99: 799-801.

10 Urakami Y, Takenaka I, Nakamura M, Fukuyama $H$, Aoyama K, Kadoya T. The reliability of the Bellhouse test for evaluating extension capacity of the occipitoatlantoaxial complex. Anesth Analg 2002; 95: 1437-41.

11 Ordway NR, Seymour RJ, Donelson RG, Hojnowski LS, Edwards WT. Cervical flexion, extension, protrusion, and retraction. A radiographic segmental analysis. Spine 1999; 24: 240-7.

12 Horton WA, Fahy L, Charters P. Disposition of cervical vertebrae, atlanto-axial joint, hyoid and mandible during x-ray laryngoscopy. Br J Anaesth 1989; 63: 435-8.

13 Panjabi M, Dvorak J, Duranceau J, et al. Three-dimensional movements of the upper cervical spine. Spine 1988; 13: 726-30.

14 Isono $S$. Common practice and concepts in anesthesia: time for reassessment: is the sniffing position a "gold standard" for laryngoscopy? (Editorial). Anesthesiology 2001; 95: 825-7. 